\title{
Physiotherapy Management of Young Male with Severe Symptoms of COVID- 19 Infection: A Case Study
}

\author{
Gauri Wakde ${ }^{1}$, Prajakta Patil ${ }^{2}$, Sumit Jadhav ${ }^{3}$, Zahara Polen ${ }^{4}$, \\ Pranali Shamkure ${ }^{5}$ \\ ${ }^{1,5}$ MPT Cardiovascular and Respiratory Physiotherapy, Smt. Kashibai Navale College of Physiotherapy, Narhe, \\ Pune, India. \\ ${ }^{2}$ Professor and HOD, Cardiovascular and Respiratory Physiotherapy, Smt. Kashibai Navale College of \\ Physiotherapy, Narhe, Pune, India. \\ ${ }^{3}$ Postgraduate Student, Smt. Kashibai Navale College of Physiotherapy, Narhe, Pune, India. \\ ${ }^{4}$ Assistant Professor, Cardiovascular and Respiratory Physiotherapy, Smt. Kashibai Navale College of \\ Physiotherapy, Narhe, Pune, India.
}

Corresponding Author: Gauri Wakde

\begin{abstract}
COVID-19 is worldwide pandemic affecting every age group with symptoms ranging from asymptomatic or mild to severe respiratory failure. COVID being highly transmittable and pathogenic disease has caused huge burden worldwide for more than a year. Severe symptoms of COVID19 require immediate hospitalisation and prompt medical management. This case study illustrates the recovery of a young male patient with severe symptoms of COVID-19 with complications of ARDS and Polyneuropathy. Physiotherapy has been an integral part of multidisciplinary team in management of COVID 19 patients especially with severe respiratory illnesses. The case report mainly highlights the Physiotherapy management for the patient in camaraderie to the Medical management which resulted in remarkable recovery of the patient.
\end{abstract}

Keywords- COVID, Coronavirus, case report, case study, severe symptoms, physiotherapy, ARDS, polyneuropathy.

\section{INTRODUCTION}

Coronavirus

disease $\quad 2019$

(COVID19) a worldwide pandemic caused by SARS-COV2, is an unprecedented global public health crisis. First case of COVID-19 was reported on 17 November, 2019 in
Wuhan, China. (1) On 30th January, the WHO declared the outbreak a Public Health Emergency of International Concern (PHEIC). ${ }^{(2)}$

Coronavirus disease is a highly transmittable and pathogenic disease which causes rapid human to human transmission having currently 140 million cases over the globe with 14.5 million cases in India with the numbers rising rapidly.(As of $16^{\text {th }}$ April 2021)

Infectious agent may transfer infection to susceptible individual directly when the person comes in contact (within 1.8 meters) with an infected symptomatic or asymptomatic individual. (3) Direct transmission is transmitted via various routes, of which aerosols, droplets and fomites became vital routes of transmission. (4) Infected individual transfers viral particles predominantly during coughing, sneezing, talking and breathing. ${ }^{(5)}$

Indirect contact with infectious secretions transferred on fomites can cause the transmission. These aerosols are active in vicinity air for 3 hours which subsequently become fomites. ${ }^{(6)}$ The people confined to these surroundings are at risk of viral transmission by use of infected objects and surfaces. Virus particles remain active 
on plastic for up to 2-3 days, stainless steel for up to 2-3 days, cardboard for up to 1 day, copper for up to 4 hours. ${ }^{(7)}$

The incubation period for COVID 19 is 3-7 days (median 5.1 days) and up to 2 weeks as longest form of infection was $12.5 / 11.5$ days in $97.5 \%$ of individuals. Various symptoms of COVID 19 include fever/chills (89\%), cough (68\%), fatigue (38\%), sputum production (34\%), dyspnoea (19\%), muscle/ body aches, and headache, new loss of taste or smell, sore throat, congestion/runny nose, nausea/vomiting, diarrhoea.COVID-19 predominantly affects the lungs. However other body systems may also be affected causing cardiac, haematological, neurological, Gastrointestinal, renal and liver dysfunctions. ${ }^{(8,9)}$ Patients with comorbidities like hypertension, diabetes, obesity, cardiovascular disease, lung disease, immunocompromised diseases have risk of severe infection to COVID 19. ${ }^{(10)}$

The radiological findings are airspace opacities, described as consolidation or ground glass opacities (GGO). The distribution is most often bilateral, peripheral, and lower zone predominant. CT Scans commonly show GGO's, crazy paving appearance, consolidation, pleural thickening, traction bronchiectasis particularly bilateral, peripheral and at bases of the lungs. ${ }^{(11)}$

The mainstay of clinical treatment consists of symptomatic management along oxygen therapy or mechanical ventilation for patients if indicated and physiotherapy.

Physiotherapy is an integral part of the multidisciplinary ICU team supporting the holistic treatment of patients and working for various aspects in COVID 19. ${ }^{(12)}$ COVID 19 patients usually present with a debilitated physical condition due to fever, fatigue, myalgia which further reduces their exercise capacity. Such devitalizing conditions can also result in prolonged immobilization and subsequently indicate mechanical ventilation if the condition worsens. Patients with moderate severity of COVID 19 also may require prolong hospitalization because of significant decrease in their activity levels. These patients are thus prone to a reduction in their muscle strength and cardiorespiratory endurance. ${ }^{(13)}$ Physiotherapy in the critical phase works mainly to wean patients off ventilator, initiates early mobilization and prevents complications which gradually progresses to regaining of strength and recovery to ADLs to improve quality of life. (14) Physiotherapist provide respiratory care and treatment which mainly comprises of postural correction, positioning, breathing exercises, dyspnoea relieving positions, limb mobility, relaxation exercises, and ambulation of patients.

The aim of this article is to share the Physiotherapy interventions and its vital importance and benefits in the management of a critical patient with severe disease of COVID-19. The patient in this case study has a diverse presentation and showed considerable improvement in response to physiotherapeutic interventions.

\section{CASE PRESENTATION}

A 30 year old male with no known comorbidities was admitted in COVID-19 suspect ward on 14/11/2020. He had no history of contact with COVID 19 patients nor had history of travel. He came with chief complaints of breathlessness (MMRC 4), dry cough, fever, fatigue, loss of taste since 2 days.

On general examination, patient was febrile with fever of $101 \mathrm{~F}$, with pulse rate $125 \mathrm{bpm}$ and blood pressure 135/86 mm Hg. On auscultation, air entry was bilaterally reduced with presence of fine crackles over middle and lower zones bilaterally. Physical examination revealed tachypnoea (34 breaths/minute), nasal flaring, increased work of breathing, and use of accessory inspiratory muscles. Patient's ABG report immediately after admission revealed hypoxaemia, hypercapnia with type 1 respiratory failure and severe ARDS. ${ }^{(15)} \mathrm{X}$ ray revealed overall haziness, obliteration of cardio phrenic and costo-phrenic angles and fibrotic changes. (Image 1) Chest CT 
revealed subpleural thickening, GGO's, honeycombing appearances. The patient's relatives were explained about the condition and counselled.

His RT-PCR test for COVID-19 came positive on 15th November 2020 after which he was hospitalized in COVID ward in tertiary Care Hospital in Pune. At the time of admission his SPO2 was $75 \%$ on room air which improved to $89 \%$ on administering $\mathrm{O} 2(9 \mathrm{~L} / \mathrm{min})$ by simple oxygen mask.

On $16^{\text {th }}$ November patient had sudden drop in saturation SPO2 55\%, with tachypnoea $50 \mathrm{bpm}$, tachycardia $150 \mathrm{bpm}$ along with gasping and increased work of breathing. Patient was shifted to COVID-19 ICU where emergency intubation was done. Patient was kept on volume AC mode of ventilator with Fio2 of $100 \%$.

The patient's condition continued to be stagnant and hence elective Tracheostomy was done on $1 / 12 / 2020$. His RTPCR was negative on 4/12/2020. However he still required ventilator support on volume $\mathrm{AC}$ with $60 \%$ Fio2 hence was shifted to non COVID MICU on 5/12/2020

When the patient came to MICU he was on vol control $50 \%$ fio2, with HR 120 bpm, SPO2 90\%, RR 25 bpm. While patient was on ventilator he gradually started experiencing weakness in his bilateral lower limbs. NCV examination was done on 22/12/2020 which suggested axonal sensorimotor polyneuropathy.

Since the patient started responding better and had significant improvement he was shifted to HDU on $5^{\text {th }}$ January on 11/L/min O2 via Non rebreathing mask. The exercises were progressed gradually to active in bed limb and breathing exercises. Subsequently patient was taken to bedside sitting whereby strengthening activities and independent sitting was focused. When patient started tolerating sitting position, progression was made to standing position. Initially there was drop in saturation from $92 \%$ to $85 \%$, along with tachypnoea which improved when patient was given sit to stand regularly.
After a 3-4 days supported sit to stand, standing was attempted. After rigorous physiotherapy patient progressed to independent standing and spot marching. During this patient was simultaneously trained for activity pacing and ambulating 23 steps. The distance of ambulation was progressed gradually.

When the patient started tolerating standing position and maintaining normal vitals in standing, progression to trunk rotation, squats (5-10 repetitions) was made. Meanwhile all the previous breathing, mobility and strengthening exercises continued. As per the guidelines, drop of oxygen saturation $>3$ percent and/or increase in the pulse rate above 120 beats per minute was considered as terminating criteria for exercise to restore the oxygen saturation and pulse rate until the vitals stabilize. ${ }^{(12,16)}$

Investigations performed are mentioned in (Table 1). ABG reports are presented in (Table 2)

\begin{tabular}{|l|l|l|}
\hline Investigations & \multicolumn{2}{|c|}{ Interpretations } \\
\hline Investigations & Heart rate-125beats per & $\begin{array}{l}\text { Suggestive of } \\
\text { Right } \\
\text { minute. PVCs present } \\
\text { intermittently in V1, V2. }\end{array}$ \\
\hline Haemiation
\end{tabular}


Gauri Wakde et.al. Physiotherapy management of young male with severe symptoms of COVID- 19 infection: a case study.

\begin{tabular}{|c|c|c|c|c|c|}
\hline Date & $15 / 11 / 2020$ & $21 / 11 / 2020$ & $28 / 11 / 2020$ & $06 / 12 / 2020$ & $15 / 12 / 2020$ \\
\hline Day of admission & Day $2^{\text {nd }}$ & Day 8th & Day $15^{\text {th }}$ & Day 23rd & Day 32nd \\
\hline $\mathrm{O} 2$ status & 9 lit via FM & $\begin{array}{l}\mathrm{MV} \text { on } \mathrm{Vol} \mathrm{A} / \mathrm{C} \text { on } \\
100 \% \mathrm{FiO} 2\end{array}$ & $\begin{array}{l}\mathrm{MV} \text { on } \mathrm{Vol} \mathrm{A} / \mathrm{C} \text { on } \\
90 \% \mathrm{FiO} 2\end{array}$ & $\begin{array}{l}\mathrm{MV} \text { on } \mathrm{Vol} \mathrm{A} / \mathrm{C} \text { on } 60 \% \\
\mathrm{FiO} 2\end{array}$ & $\begin{array}{l}\text { CPAP on } 60 \% \\
\mathrm{FiO} 2\end{array}$ \\
\hline $\mathrm{pH}$ & 7.33 & 7.30 & 7.40 & 7.426 & 7.435 \\
\hline $\mathrm{pCO} 2(\mathrm{mmHg})$ & 50 & 52.3 & 49.6 & 46.5 & 42.6 \\
\hline $\mathrm{HCO} 3(\mathrm{mmol} / \mathrm{L})$ & 20 & 19 & 21.5 & 30.5 & 25.7 \\
\hline $\mathrm{pO} 2(\mathrm{mmHg})$ & 70 & 62.9 & 73.8 & 79.3 & 82.1 \\
\hline SPO2 & 89 & 84 & 89 & 92 & 94 \\
\hline $\mathrm{PaO} 2 / \mathrm{FiO} 2$ & 100 & 85 & 130 & 170 & 230.5 \\
\hline Date & $25 / 12 / 2020$ & $31 / 12 / 2020$ & $3 / 01 / 021$ & $08 / 01 / 2021$ & $22 / 1 / 2021$ \\
\hline Day of admission & Day $42^{\text {nd }}$ & Day 48th & Day 51st & Day $56^{\text {th }}$ & Day 70th \\
\hline O2 status & $\begin{array}{l}7 \text { lit via } \mathrm{T}- \\
\text { piece }\end{array}$ & $\begin{array}{l}15 \mathrm{~L} / \mathrm{min} \\
\mathrm{NRBM}\end{array}$ & $\begin{array}{l}11 \mathrm{~L} / \mathrm{min} \\
\mathrm{NRBM}\end{array}$ & $\begin{array}{l}6 \mathrm{~L} / \mathrm{min} \\
\text { Via face mask }\end{array}$ & Room Air \\
\hline $\mathrm{pH}$ & 7.45 & 7.47 & 7.50 & 7.52 & 7.48 \\
\hline $\mathrm{pCO} 2(\mathrm{mmHg})$ & 40.6 & 39.4 & 35.7 & 33.4 & 39.3 \\
\hline $\mathrm{HCO} 3(\mathrm{mmol} / \mathrm{L})$ & 25.6 & 24.1 & 22.7 & 23.1 & 22.4 \\
\hline $\mathrm{pO} 2(\mathrm{mmHg})$ & 73.6 & 79.4 & 83.9 & 89.4 & 90.8 \\
\hline SPO2 & 96 & 96 & 97 & 98 & 98 \\
\hline $\mathrm{PaO} 2 / \mathrm{FiO} 2$ & 250 & 255.3 & 280 & 310 & 320 \\
\hline
\end{tabular}
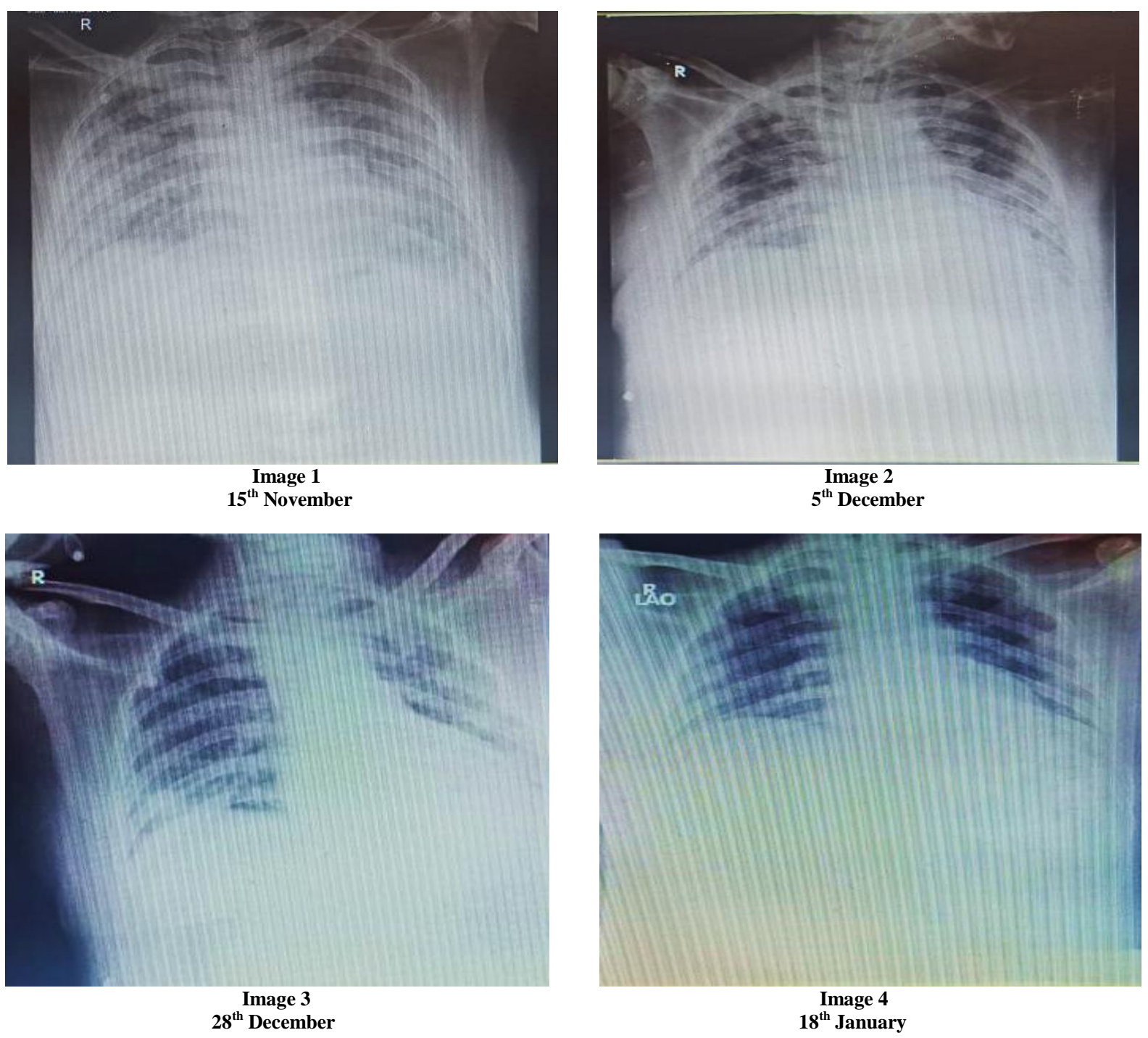

\section{DISCUSSION}

COVID 19 is a worldwide pandemic which began in December 2019 in China which has now spread tremendously all over

the globe infecting the world for more than a year. Due to its massive destruction and devastation around the world, there are various treatment strategies being analysed 
and implemented. Even today inspite of vaccine being available, the management mainly focuses on symptomatic treatment with root cause being untouched. Constant bombardment with news regarding rapidly increasing rate of infections and deaths every day, shortage of medications, sharing of controversial messages and videos over social media, multiple lockdown strategies have led to anxiety, panic and fear amongst common people. Inspite of initiate efforts from dawn to dusk the situation has led to low faith of common people in the health care system developing social stigma and taboo against the people affected and even recovered with COVID-19.The reluctance and negligence of people to mild symptoms worsens the prognosis. The patients often report to hospitals after deterioration with severe symptoms. ${ }^{(10)}$

The case highlights successful treatment of the patient with severe symptoms through holistic approach by medical management, Physiotherapy management, Psychological counselling along with Nutrition of the patient which led to life from death bed, from ventilator to breathing spontaneously and walking independently from paresis. Such successful treatment of the patient despite having severe symptoms helps to restore faith and instil confidence amongst common people about the health care system and encourage doctors to work more efficiently.

The patient was referred for Physiotherapy on the same day after admission. Patient was given required medications, was ventilated along with healthy nutritious diet in the hospital. Day 2 of hospitalization patient had sudden drop in saturation for which emergency intubation was done. Patient was on volume AC mode with Fio 2 of $100 \%$. The Fio2 requirements were consistent to $70-100 \%$ for around 1315 days. During this period airway management, suctioning, positioning in lateral and propped up, chest PNF, passive/active assisted limb mobility was given. The treatment was given according to the prescribed guidelines for physiotherapy practice in COVID 19. Patient's reference heart rate and oxygen saturation were continuously monitored during each exercise using pulse oximeter.

Subsequent $\mathrm{ABG}$ reports revealed improvement in $\mathrm{PaO} 2$, ventilation perfusion mismatch, A/a gradient. (17) Patient was taken on CPAP on $15^{\text {th }}$ December and gradually on $\mathrm{T}$ piece by $25^{\text {th }}$ December.

However patient started developing weakness in bilateral lower limbs. The strength was reduced to $2+$ for both the lower limbs. NCV was performed on $29^{\text {th }}$ December whereby patient was diagnosed with sensorimotor polyneuropathy. Hence rigorous limb physiotherapy was started along with chest physiotherapy where active assisted movements of lower limbs, joint compression techniques, balance training along with strengthening activities were given. Exercises were progressed depending on RPE. The vitals were maintained within normal limits during the exercises with help of adequate rest intervals between exercises.

On $20^{\text {th }}$ Jan 2021, significant improvement in air entry was seen bilaterally. Physiotherapy intervention along with medical management helped in improving ventilation perfusion mismatch, alveolar ventilation optimizing oxygen transport and in maintaining normal fluid distribution in the body. It also helped to prevent complications of bed rest, deconditioning and DVT. (18, 19) The physiotherapy management in parallel with medical management, nutritional care and counselling of the patient ensured excellent recovery, demonstrating improvement in the subsequent X-ray and ABG report, bilateral fluffy shadows seen indicative of resolving ARDS. (Image 4) NCV also showed significant improvement. The patient was able to walk independently with minimum support. The patient was able to maintain normal oxygen saturation $(98 \%)$ on room air.

The patient was discharged on $25^{\text {th }}$ January after all his vitals were stable and patient was able to walk independently. 
Gauri Wakde et.al. Physiotherapy management of young male with severe symptoms of COVID- 19 infection: a case study.

Two months of rigorous team work of physiotherapy treatment along with the medical management worked fantastically for the patient, manifesting importance of physiotherapy in COVID and also imbibed the importance of team work and boosted courage amongst all the doctors during these tough times.

Table 3- changes in vital parameters with inpatient supervised physiotherapy management

\begin{tabular}{|c|c|c|c|c|c|c|c|}
\hline Physiotherapy treatment day & $20-11-2020$ & \multicolumn{2}{|c|}{$21-11-2020$} & $26-11-2020$ & 01-12-2020 & $02-12-2020$ & 08-12-2020 \\
\hline O2 supply & $\begin{array}{r}\mathrm{MV} \text { on } \mathrm{Vol} \mathrm{A} / \mathrm{C} \\
100 \% \mathrm{FiO}_{2} \\
\end{array}$ & \begin{tabular}{r|r} 
on & MV on $V_{c}$ \\
$80 \%$ \\
\end{tabular} & \begin{tabular}{l|r}
$\mathrm{A} / \mathrm{C}$ on & $\mathrm{M}$ \\
$\mathrm{FiO} 2$ & 0 \\
\end{tabular} & \begin{tabular}{c|} 
MV on $\mathrm{Vol} A / \mathrm{C}$ \\
on $90 \% \mathrm{FiO} 2$
\end{tabular} & Tracheostomy & $\begin{array}{c}\text { Vol A/C on } \\
60 \% \mathrm{FiO} 2\end{array}$ & $\begin{array}{c}\text { CPAP on } 60 \% \\
\text { FiO2 } \\
\end{array}$ \\
\hline Pulse Rate & 88 bpm & 114 & $\mathrm{pm}$ & $100 \mathrm{bpm}$ & $106 \mathrm{bpm}$ & $95 \mathrm{bpm}$ & 84 bpm \\
\hline $\mathrm{SpO}_{2}$ & $90 \%$ & 89 & & $92 \%$ & $90 \%$ & $94 \%$ & $95 \%$ \\
\hline Positioning (side lying) & $\mathrm{Y}$ & Y & & Y & $Y$ & $Y$ & $\mathrm{Y}$ \\
\hline Prone lying & Y & Y & & Y & Y & Y & Y \\
\hline Breathing Exercises & & & & & & & Y \\
\hline \multicolumn{8}{|l|}{ Incentive Spirometer } \\
\hline In-bed Exercises & & & & & & & Y \\
\hline \multicolumn{8}{|l|}{ Bedside sitting exercises (without support) } \\
\hline Chest PNF & Y & Y & & Y & Y & Y & \\
\hline Passive ROM Exs & Y & Y & & Y & $Y$ & Y & \\
\hline Chest PT & Y & Y & & Y & Y & Y & Y \\
\hline Active-Assisted ROM Exs & & & & & & Y & $\mathrm{Y}$ \\
\hline \multicolumn{8}{|l|}{ Bedside sitting exercises (with support) } \\
\hline \multicolumn{8}{|l|}{\begin{tabular}{|l|} 
Postural Correction \\
\end{tabular}} \\
\hline \multicolumn{8}{|l|}{ Gluteal Strengthening } \\
\hline \multicolumn{8}{|l|}{ Back Strengthening } \\
\hline \multicolumn{8}{|l|}{ TA stretching } \\
\hline \multicolumn{8}{|l|}{ VMO strengthening } \\
\hline \multicolumn{8}{|l|}{ Dorsiflexors Strengthening } \\
\hline \multicolumn{8}{|l|}{ Standing } \\
\hline \multicolumn{8}{|l|}{ Wall Squats } \\
\hline \multicolumn{8}{|l|}{ Spot Marching } \\
\hline \multicolumn{8}{|l|}{ Ambulation } \\
\hline Physiotherapy treatment day & $15-12-2020$ & $22-12-2020$ & $29-12-2020$ & 06-01-2021 & $13-01-2020$ & 20-01-2021 & 24-01-2021 \\
\hline O2 supply & $\begin{array}{c}\text { CPAP on } 40 \% \\
\text { FiO2 } \\
\end{array}$ & T-PIECE & NRBM & NRBM & FM & Room air & Room air \\
\hline Pulse Rate & 76 bpm & $89 \mathrm{bpm}$ & 112 bpm & 107 bpm & $99 \mathrm{bpm}$ & $87 \mathrm{bpm}$ & $85 \mathrm{bpm}$ \\
\hline $\mathrm{SpO}_{2}$ & $97 \%$ & $98 \%$ & $95 \%$ & $94 \%$ & $98 \%$ & $95 \%$ & $98 \%$ \\
\hline Positioning (side lying) & $\mathrm{Y}$ & $\mathrm{Y}$ & $\mathrm{Y}$ & $\mathrm{Y}$ & $\mathrm{Y}$ & $\mathrm{Y}$ & $\mathrm{Y}$ \\
\hline Prone lying & Y & $\mathrm{Y}$ & Y & $\mathrm{Y}$ & $\mathrm{Y}$ & $\mathrm{Y}$ & $\mathrm{Y}$ \\
\hline Breathing Exercises & Y & Y & Y & Y & Y & Y & Y \\
\hline Incentive Spirometer & $\mathrm{Y}$ & Y & $\mathrm{Y}$ & Y & Y & Y & Y \\
\hline In-bed Exercises & Y & Y & Y & Y & Y & Y & Y \\
\hline Bedside sitting exercises (without support) & & & Y & Y & Y & Y & Y \\
\hline \multicolumn{8}{|l|}{ Chest PNF } \\
\hline Passive ROM Exs & & & & & & & \\
\hline Chest PT & Y & Y & & & & & \\
\hline Active-Assisted ROM Exs & Y & Y & & & & & \\
\hline Bedside sitting exercises (with support) & & Y & Y & & & & \\
\hline Postural Correction & & & Y & Y & Y & $Y$ & $Y$ \\
\hline Gluteal Strengthening & & & Y & $Y$ & Y & Y & Y \\
\hline Back Strengthening & & & Y & $Y$ & Y & Y & Y \\
\hline TA stretching & & & $\mathrm{Y}$ & Y & $\mathrm{Y}$ & $\mathrm{Y}$ & Y \\
\hline VMO strengthening & & & & $\mathrm{Y}$ & $Y$ & $\mathrm{Y}$ & $\mathrm{Y}$ \\
\hline Dorsiflexors Strengthening & & & & Y & Y & Y & Y \\
\hline Standing & & & & & Y & $Y$ & $Y$ \\
\hline Wall Squats & & & & & & $\mathrm{Y}$ & $Y$ \\
\hline Spot Marching & & & & & & $\mathrm{Y}$ & $Y$ \\
\hline Ambulation & & & & & & & $\mathrm{Y}$ \\
\hline
\end{tabular}

ABBREVIATIONS-

ABG: Arterial Blood Gas

ARDS: Acute Respiratory Distress
CARP: COVID Awake Repositioning

Protocol

EMG-Electromyography

FiO2: Fraction of Inspired Oxygen

HCO3: Bicarbonate L/min: Litres/min 
MMRC: Modified Medical Research Council

MmHg: Millimetres of mercury

Mmol/L: millimoles/Litre

NCV-Nerve conduction velocity

Pnf- Proprioceptive Neuromuscular Facilitation

PCO2: Partial Pressure of Carbon Dioxide

PaO2: Partial Pressure of Oxygen

RPE: Rate of Perceived Exertion

RT-PCR: Reverse Transcription

Polymerase Chain Reaction

SPO2: Saturation of Peripheral Oxygen

\section{Acknowledgement: None}

\section{Conflict of Interest: None}

\section{Source of Funding: None}

\section{REFERENCES}

1. Marco Cascella, Michael Rajnik, Arturo Cuomo et al: Features, Evaluation and Treatment Coronavirus (COVID-19). Stat Pearls; 2020

2. WHO: COVID-19 Public Health Emergency of International Concern (PHEIC).Global research and innovation forum; Feb 2020

3. Peter Thomas, Claire Baldwin, Bernie Bissett et al: Physiotherapy management for COVID-19 in the acute hospital setting: clinical practice recommendations. J Physiotherapy; 2020-66: 73-82.

4. Jayaweera,Hasini Perera Buddhika Gunawardana al: Transmission of COVID19 virus by droplets and aerosols. A critical review on the unresolved dichotomy; 2020

5. Transmission of SARS-CoV-2: implications for infection prevention precautions. Scientific Brief; 9 July 2020

6. Nancy $\mathrm{H}$ Leung, Transmissibility and transmission of respiratory virus; Nature Reviews Microbiology; 2020

7. NHS: Study suggests new coronavirus may remain on surfaces for days; 2020

8. Symptoms of Coronavirus: Centres for Disease Control and Prevention, 2020

9. Shima Behzad, Leila Aghaghazvini, Amir Reza Radmard et al, Extra pulmonary manifestations of COVID19: Radiologic and clinical overview; Clin Imaging ,2020;66: 35-41.

10. Revised Guidelines on Clinical Management of COVID: Government of India Ministry of Health \& Family Welfare Directorate General of Health Services (EMR Division); 2020

11. Rykiel Levine, Nicholas Caputo et al; CT scan of a COVID- positive patient, Journal of Am Physician, 2020

12. Peter Thomas, Clarie, Physiotherapy management for COVID-19 in the acute hospital setting: clinical practice recommendations. Journal of Physiotherapy; April 2020

13. Renato Fraga Righetti, Mirian Akemi Onoue et al, Physiotherapy Care of Patients with Coronavirus Disease 2019 (COVID19) - A Brazilian Experience: Sao Paulo; June 2020

14. Chhaya V Verma, Rachna D Arora, Jaimala V Shetye et al, Guidelines of physiotherapy management in acute care of COVID-19 at dedicated COVID center in Mumbai. J Indian Assoc Physiotherapy; 2020:14:55-60.

15. $\mathrm{Xu} \mathrm{Li}$, Xiaochun Ma, Acute respiratory failure in COVID-19: is it "typical" ARDS Crit Care, 2020-24: 198.

16. Guidelines for Chest Physiotherapy management of COVID 19 in Indian SetupMS OTPT Council; 2020.

17. Martin J Tobin, Basing respiratory management of COVID-19 on physiological principles. Am J Respir Crit Care Med; 2020: 201: 1319- 1320

18. Nim Pathmanathan, Nicola Beaumont et al, Respiratory physiotherapy in the critical care unit. Continuing Education in Anaesthesia, Critical Care \& Pain, 2015; 15: 20-25.

19. Juultje Sommers, Raoul H Engelbert, Daniela Dettling et al. Physiotherapy in the intensive care unit: an evidence-based, expert driven, practical statement and rehabilitation recommendations. Clin Rehabil; 2015, 29: 1051- 1063.

How to cite this article: Wakde G, Patil P, Jadhav $S$ et.al. Physiotherapy management of young male with severe symptoms of COVID19 infection: a case study. International Journal of Research and Review. 2021; 8(4): 422-428. DOI: https://doi.org/10.52403/ijrr.20210450 